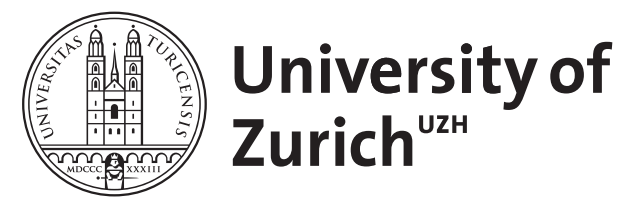

\title{
The brain of synesthetes
}

\author{
Jäncke, Lutz
}

\begin{abstract}
Synesthesia is a fascinating phenomenon in which a particular perception induces a concurrent perception resulting in a kind of double perception. This phenomenon has received considerable attraction in the last 15 years by neuroscientists and cognitive psychologists. Here I will summarize and discuss some of the main findings and ideas from this research. First, I will discuss the main neurophysiological models trying to explain this extraordinary phenomenon. Secondly, I will describe the findings trying to delineate the time course of synesthetic perception in relation to the associated neurophysiological models. Finally, current findings reporting specific and general neuroanatomical features of the synesthete's brain will be discussed. These findings will be integrated into the current models about the neurophysiological underpinnings of synesthesia.
\end{abstract}

DOI: https://doi.org/10.1007/s12210-014-0323-z

Posted at the Zurich Open Repository and Archive, University of Zurich ZORA URL: https://doi.org/10.5167/uzh-101163

Journal Article

Published Version

Originally published at:

Jäncke, Lutz (2014). The brain of synesthetes. Rendiconti Lincei. Scienze Fisiche e Naturali, 25(3):309316.

DOI: https://doi.org/10.1007/s12210-014-0323-z 


\title{
The brain of synesthetes
}

\author{
Lutz Jäncke
}

Received: 25 February 2014/ Accepted: 14 July 2014/Published online: 5 August 2014

(C) Accademia Nazionale dei Lincei 2014

\begin{abstract}
Synesthesia is a fascinating phenomenon in which a particular perception induces a concurrent perception resulting in a kind of double perception. This phenomenon has received considerable attraction in the last 15 years by neuroscientists and cognitive psychologists. Here I will summarize and discuss some of the main findings and ideas from this research. First, I will discuss the main neurophysiological models trying to explain this extraordinary phenomenon. Secondly, I will describe the
\end{abstract}

This contribution is the written, peer-reviewed version of a paper presented at the Giornata Golgi 2013 "Brain Science and Human Culture", held at the Accademia Nazionale dei Lincei in Rome on June 3rd, 2013.

Parts of this chapter have been published in a recent book chapter (Jäncke 2013). There are also some wordings, which I have used in previous publications (e.g., Jäncke et al. 2012; Jäncke and Langer 2011).

\section{Jäncke $(\bowtie)$}

Division Neuropsychology, Institute of Psychology, University of Zurich, Binzmühlestrasse 14/25, 8050 Zurich, Switzerland e-mail: lutz.jaencke@uzh.ch

\section{Jäncke}

Center for Integrative Human Physiology, University of Zurich, Zurich, Switzerland

L. Jäncke

International Normal Aging and Plasticity Imaging Center (INAPIC), University of Zurich, Zurich, Switzerland

\section{Jäncke}

University Research Priority Program (URPP) "Dynamic of Healthy Aging”, University of Zurich, Zurich, Switzerland

L. Jäncke

Department of Special Education, King Abdulaziz University, Jeddah, Saudi Arabia findings trying to delineate the time course of synesthetic perception in relation to the associated neurophysiological models. Finally, current findings reporting specific and general neuroanatomical features of the synesthete's brain will be discussed. These findings will be integrated into the current models about the neurophysiological underpinnings of synesthesia.

Keywords Synesthesia $\cdot$ FMRI $\cdot$ SMRI $\cdot$ EEG $\cdot$ Models of synethesia

\section{Introduction}

Synesthesia is a rare perceptual phenomenon in which an inducing stimulus (and the associated perception) elicits concurrent perceptions. A recent study (Niccolai et al. 2012a) has shown that most of the synesthetes experience more than one form of synesthesia (thus, they are multisynesthetes). In addition, most of the synesthetes demonstrate "Multi-modal synesthesia", in which the inducer perception and the concurrent perception belong to two different sensory modalities, e.g., vision and auditory modality). In arts synesthesia is often associated with a more common form of simultaneous perception of multiple stimuli generating a kind of "Gestalt"experience. Many famous artists have claimed to be synesthetes or others have explained their extraordinary skills with synesthetic abilities. In his book "The hidden sense: synesthesia in art and science" Campen (2010) describes several artists who used synesthesia as a kind of inspiration for their work. For example the famous painters Kandinski (who claimed to be a synesthete, although never proved) and Mondrian (who also claimed to be a synesthete, although never proved) experimented with color-tone-music associations during 
their paintings. However, in the light of modern neuroscience and cognitive science, it is more a kind of conscious and unconscious association between different senses. Several famous musicians also explained their work on the basis of synesthetic experiences (e.g., Scriabin and Messiaen). On Wikipedia (http://en.wikipedia.org/wiki/Synes thesia) several artists are listed as synesthetes among them Arthur Rimbaud, Charles Baudelaire, Duke Ellington, Vladimir Nabakov, or Nikolai Rimsky-Korsakov to name only a few. However, as mentioned above, there is no scientific proof whether they are "true" synesthetes or whether they are only subjects who are able to associate different sensory modalities more efficiently than ordinary people. On a scientific basis, synesthesia is currently defined "as a neurological phenomenon in which stimulation of one sensory or cognitive pathway leads to automatic, involuntary experiences in a second sensory or cognitive pathway" (Simner 2012). Associations (between thoughts, perceptions, and memory contents) are, in contrast to synesthetic experiences, mostly conscious, relatively slow, are deliberately evoked, and can be inhibited.

The term synesthesia was first used by the French neurophysiologist Vulpian (1866). But the Swiss psychiatrists Bleuler and Lehmann (1871) were the first to conduct sophisticated investigations of this particular phenomenon. Bleuler's and Lehmann's interests in synesthesia were mainly driven by the goal of understanding hallucinatory experiences especially in schizophrenia. A better understanding of the psychological, neurophysiological, and neuroanatomical underpinnings of synesthesia itself failed thereafter to attract research interest. But with the advent of modern neurophysiological registration methods, a growing community of neuroscientists is engaged in the studying of synesthesia (Rouw et al. 2011). An important milestone was the first brain imaging study of Paulesu et al. (1995) who used positron emission tomography to measure hemodynamic responses in synesthetes during synesthetic experiences. Several other studies followed using functional magnetic resonance imaging to explore the hemodynamic responses in synesthetes (Weiss et al. 2001, 2005; Nunn et al. 2002; Elias et al. 2003; Hubbard et al. 2005; van Leeuwen et al. 2010). In general, these studies show that the concurrent perception is associated with hemodynamic responses in brain areas that are normally involved in processing the stimuli perceived during synesthetic experience. Thus, in colored-hearing synesthetes, a tone induces activations in the auditory cortex and in the fusiform color area.

Alongside these first brain imaging studies, event-related potentials (ERP) are more and more used allowing the delineation of the time course of neurophysiological activations associated with synesthetic experiences (Barnett et al. 2008a, b; Beeli et al. 2008; Brang et al. 2008, 2010;
Cohen Kadosh et al. 2007; Gebuis et al. 2009; Goller et al. 2009; Niccolai et al. 2012b; Schiltz et al. 1999; Teuscher et al. 2010). Based on these experiments, the neurophysiological underpinnings of synesthesia may be described and discussed in terms of three basically different neurophysiological models: (1) the two-stage cross-activation and hyper-binding model (Hubbard 2007), (2) the disinhibited feedback model (Grossenbacher and Lovelace 2001), and (3) the limbic-mediation model (Cytowic and Wood 1982a, b). I will briefly describe these models because they make different predictions about the time course of synesthetic experiences, the underlying neurophysiological processes and the associated neuroanatomical features.

\section{Neurophysiological models}

The cross-activation and hyper-binding model was proposed on the basis of fMRI studies in grapheme-color synesthetes (Hubbard 2007). This model has been conceptualized on the basis of the relatively frequently occurring and examined grapheme-color synesthesia. According to this model, the grapheme (posterior temporal grapheme area: PTGA) and color processing brain areas (V4) are thought to be aberrantly connected. A key idea behind this is that cross-activation between these brain areas occurs because of an abnormal excess of anatomical connections between the grapheme and color processing brain areas. This aberrant connectivity should result in strong co-activation of these areas during grapheme processing under the control of networks located in the vicinity of the intraparietal sulcus (the latter process resulting in hyper-binding). This model has received some support, specifically for experience of auditory-color synesthesia (e.g., Jäncke and Langer 2011).

The disinhibited feedback model is based on studies demonstrating specific forms of acquired and congenital synesthesias rather than on data from brain imaging studies (Grossenbacher and Lovelace 2001). The disinhibited feedback model (also basing on ideas mainly motivated by grapheme-color synesthesias) diverges from the crossactivation and hyper-binding model in that it posits normal connectivity patterns in synesthetes. It also suggests that synesthesia results from disinhibited feedback from higherlevel cortical areas in the visual processing hierarchy. This loss of top-down inhibition should cause a kind of overactivation of the subordinate brain areas. A hybrid model, the so-called re-entrant processing model, shares with the cross-activation model the notion of hyper-connectivity between form and color processing areas in the fusiform area, and suggests, like the disinhibited feedback model, that synesthetic colors require feedback of neural activity 
that originates in higher-level areas (e.g., anterior inferior temporal and posterior inferior temporal) to V4 (Smilek et al. 2001).

The limbic-mediation hypothesis, first proposed by Cytowic and Wood (1982a, b) which is mainly based on theoretical reflections and not on empirical data, proposes that synesthesia is mediated by the limbic system (and especially the hippocampus), on which multiple sensory signals converge. According to this hypothesis, synesthetes should have more connective fibers leading from the limbic system to the neocortex. Based on this limbic-neocortex hyper-connectivity, Cytowic and Wood propose that synesthetes are more aware or conscious of functional crossmodal couplings. Thus, the notion of a kind of enhanced cross-modal coupling is pivotal to this theory. Interestingly, this model does not make any assumptions about the time course of synesthetic experiences and the associated neurophysiological activations.

Different predictions can be drawn from these models about the timing of neural activations. On the basis of the cross-activation and hyper-binding model, there should be a particular time lag between the processing of the inducing stimulus (the inducer) and the concurrent perception. Thus, the neurophysiological activation of the brain areas associated with processing of the concurrent perception should follow with a significant delay the activation of the brain areas associated with the processing of the inducing stimulus. The cross-activation and hyper-binding model also suggests increased local connectivity and predicts the activation of V4 during the initial sweep of activity in the posterior temporal grapheme areas (PTGA) (Ramachandran and Hubbard 2001). Activation of V4 and PTGA should therefore occur more or less simultaneously. The limbic-mediation hypothesis does not make any assumptions about the timing of the neurophysiological processes. However, since the limbic system and the neocortex are strongly interconnected and the limbic system is involved in simple and complex cognitive and sensory processes fast and slow processes are possible. Thus, even when hyperconnectivity between limbic and neocortical systems would exist, activation time lags between the involved brain areas are possible but not necessary. In terms of the hypothesized anatomical features one would anticipate that synesthetes should show stronger anatomical connectivity between those brain areas, which are involved in synesthetic experiences. In addition, the brain areas involved in synesthetic experiences should show some particular anatomical features, which are associated with superior processing (e.g., increased gray matter density or changed cortical thickness).

Although these models are used as starting points for many current neurophysiological and psychological studies on synesthesia, it has to be kept in mind that these models have mainly been motivated by the frequently occurring grapheme-color synesthesia. Whether these models are also useful to explain the neural underpinnings of other forms of synesthesia has to be shown.

While the neural underpinnings of synesthesia are currently studied quite intensively, it is more or less unknown whether synesthesia is genetically determined, due to some kind of unknown specific maturation processes, or due to neurological disturbances. Until now there is no definitive prove for a genetic origin of synesthesia. However, there is strong evidence that synesthesia tends to run in families, as approximately $40 \%$ of synesthetes report a first-degree relative with the condition (Baron-Cohen et al. 1996; Barnett et al. 2008a, b). In at least one confirmed instance, synesthesia is present in only one monozygotic twin (Smilek et al. 2002, 2005). In their recent theoretical paper about the possible genetic origin of synesthesia Brang and Ramachandran (2011) suggest on the basis of a pedigree analysis of synesthesia high transmissibility from parent to offspring. Although the genetic origin has not been proven so far, Brang and Ramachandran (2011) argue that synesthesia is a highly heritable phenomenon, which is associated with numerous cognitive benefits, which might be the reason why this condition has survived evolutionary pressures.

\section{Time course of activation in synesthetes}

The analysis of the time course of neurophysiological activations during synesthetic experience offers the opportunity to directly test some of the assumptions associated with the three above-mentioned models. In this context, it can be tested whether synesthetic experiences are driven by early perceptual (bottom-up) or later more cognitive processing steps (top-down), or even by an interaction of these earlier and later processes.

EEG and magnetencephalographic (MEG) methods have been used to delineate the time course of neurophysiological activation during synesthetic perceptions because they allow the measurement of cortical activations on a millisecond basis. Two types of studies have thus far used these techniques in synesthesia research: the first used measures of evoked potentials time-locked to the inducing stimuli (Schiltz et al. 1999; Goller et al. 2009; Brang et al. 2008, 2010; Beeli et al. 2008; Jäncke et al. 2012; Volberg et al. 2013; Sinke et al. 2014), which have been either visual (e.g., graphemes for grapheme-color synesthetes) or auditory stimuli (tones or words for auditory-color graphemes), and the second type used priming techniques and measured higher-order cognitive processes in the context of synesthetic experience (Gebuis et al. 2009; Niccolai et al. 2012b). 
Most of these studies have shown that synesthetes demonstrate different neurophysiological reactions quite early in the processing stream happening around 80-150 ms after presentation of the inducer. The neurophysiological differences even extend into later processing stages up to 300-400 ms after stimulus presentation. Thus, synesthetes obviously process inducers differently for a quite long time but the differences appear quite early at processing stages during which conscious processing is not likely. A typical example of these studies is a recent study by our group (Jäncke et al. 2012). In this study, we used a mismatch negativity (MMN) paradigm to examine preattentive mechanisms in the context of tone-color synesthetic perceptions. In the context of this MMN paradigm, the subjects were exposed to tones of different frequencies while they watched a silent black and white movie in the forefront. The tones are presented in the background in rapid succession most of the tones are similar in terms of frequency (the standards) while some few deviate from the standard in terms of frequency (the deviants). In the context of this paradigm, the event-related potentials (ERPs) to the standards and deviants are subtracted to obtain the difference ERP, which shows the MMN a negative going wave 100-150 ms after stimulus onset. The amplitude of this MMN is larger the better the subjects are able to preattentively discriminate standards and deviants. Synesthetes consistently showed larger MMN responses than non-synesthetes. Thus, they obviously discriminate preattentively tones much more efficiently than non-synesthetes. The intracerebral sources of this MMN were spatially widespread in the synesthetes comprising bilaterally the entire perisylvian brain region (with the primary and secondary auditory cortex) extending into the inferior parietal cortex as well as the inferior temporal cortex. In non-synesthetes, there was only a relatively focal intracerebral source in perisylvian brain areas being slightly larger in the right hemisphere. Taken together during tone presentation several brain regions are simultaneously activated in synesthetes even when they do not actively and attentively listen to these tones. Thus, 100-150 ms after tone presentation, we identified a distributed network, which was differently activated in these tone-color synesthetes compared to non-synesthetes. This network comprises brain areas, which have previously been identified to be involved in synesthetic experiences, like the parietal and the extra-striate cortex. Furthermore, since a major source of the MMN was estimated in the auditory cortex (primary and secondary areas), it is possible that the coupling between auditory and visual experiences is driven by brain activity originating from the auditory-related cortex. Beside these strong differences for the MMN, we also identified strong P3a responses at frontal brain areas for the synesthetes. The larger P3a component indicates that the bottom-up attention network is stronger activated in synesthetes. We interpret these findings as a neurophysiological indicator of a more efficient and stronger reorienting of attention to the tone stimuli. The intracerebral sources of the P3a are located in the left-sided posterior superior and middle temporal gyrus, and at the posterior end of the superior temporal sulcus in the vicinity of the temporoparietal junction. In addition, we observed a spot of stronger current density in the right-sided inferior parietal cortex in $\mathrm{CH}$ synesthetes. These brain regions are known to be engaged in the perception of complex sounds (Oechslin et al. 2010), audiovisual processing of speech stimuli, and audiovisual integration in general (Bunzeck et al. 2005; Jäncke and Shah 2004; Proverbio et al. 2011; Schiltz et al. 1999).

A more recent study showed that synesthetic experiences are associated with short- and long-range functional connectivities (Volberg et al. 2013) also supporting the idea that early and late cognitive processes are involved in generating and controlling synesthesia. The authors of this study used high-density EEG and measured neural synchrony in a sample of grapheme-color synesthetes that were presented with color-inducing versus non-colorinducing graphemes. They detected a decrease in the number of long-range couplings and a concurrent increase of short-range phase-locking. They argue that the early and local increases of neural synchrony support the idea of an early cross-wiring between brain areas associated with the generation of synesthesia. The later and broader pattern of neural synchrony is in favor of the idea that synesthetic color sensations rely on vertical long-range communication, which may indicate a kind of reduced inhibition in processing stages, which integrate different information coming from different modalities. These results nicely fit to findings demonstrating that the brain of synesthetes demonstrates a different functional connectivity even at resting state, with stronger connectivity in brain areas involved in local and distant processing of synesthetic experiences (Jäncke and Langer 2011). Several further ERP and behavioral studies also supported the view that synesthetic experiences are controlled by an interaction between early (more perceptual) and later (more cognitive) processes (Gebuis et al. 2009; Brang et al. 2008; Cohen Kadosh et al. 2007; Teuscher et al. 2010; Niccolai et al. 2012b).

Thus, these studies demonstrate that neurophysiological interactions occur between different processing stages in synesthetes. This idea fits well with studies examining whether bidirectional influences can be found during synesthetic experiences. Synesthesia is mostly described as unidirectional with a particular inducer triggering a particular concurrent perception. However, the concurrent does not trigger the experience of its inducer. Several behavioral studies have challenged this view by 
demonstrating back-and-forth associations between inducer and concurrent perceptions for digits and colors as well as graphemes and colors (Knoch et al. 2005; Meier and Rothen 2007; Weiss et al. 2009). These findings are consistent with earlier suggestions proposing that perceptual and cognitive mechanism are involved in synesthesia (Ramachandran and Hubbard 2001; Smilek et al. 2002). Ramachandran and Hubbard distinguished "higher" synesthetes' from "lower" synesthetes while Smilek and Dixon have used the terms "projectors" and "associators". In higher/ associators synesthetes, the concept of the inducer is thought to be critical for eliciting synesthetic experiences while in lower/projectors synesthetic experiences are more strongly driven by perceptual bottom-up processes. Whether these different processing strategies are active within all synesthetes or whether these processing strategies are more or less simultaneously used in all synesthetes has to be shown in future experiments.

\section{Neuroanatomy of the synesthete's brain}

With the advent of modern brain imaging techniques several studies have been conducted so far examining the specific features of the synesthetes brain. Using functional magnetic resonance imaging (fMRI and PET) techniques it has been shown that the sensory brain areas corresponding to the type of synesthetic experience are activated. Thus, synesthetic color experiences for example evoked by graphemes) can activate color region in the occipito-temporal cortex, but this activation is not restricted to V4. However, the activations even go beyond that as it has been shown that a network of brain areas is involved the control of synesthesia (Rouw et al. 2011; Jäncke and Langer 2011). ${ }^{1}$ Several brain regions have been shown to be pivotal for synesthetic experience among them are sensory and motor regions as well as so-called "higher-level" regions in the parietal and frontal lobe. These "higher-level" brain areas are most likely related to three different cognitive processes inherently part of synesthesia: the sensory processes (with the sensory areas), the attentional processes especially controlling the binding process (within the parietal lobe), and the cognitive processes (controlled by frontal brain regions). Most interestingly, these brain areas mostly demonstrate some kind of specific anatomical features as measured with structural MRI (sMRI). For example, the color areas or the areas in the vicinity of V4 often demonstrate larger gray matter density. In addition, parietal

\footnotetext{
${ }^{1}$ In the Rouw et al. (2011) review fMRI and sMRI papers are reviewed exploring the neural underpinnings of synesthetic experiences. I refrain from discussing all papers individually since they are mostly summarized in this review. I will only explicitly mention those fMRI and sMRI studies, which have been published since then.
}

brain areas (especially the intraparietal sulcus: IPS) have repeatedly been identified as brain areas with increased gray matter density in synesthetes. Some papers have also identified specific anatomical features in frontal brain areas. Thus, those brain areas, which are strongly involved in generating and controlling the synesthetic experiences, are anatomically different compared to non-synesthetes. Only few studies have used diffusion tensor imaging (DTI) to measure and reconstruct the fibers connecting the different brain areas. These few studies have shown that there are also some kinds of specific interconnections between he above-mentioned involved brain areas.

Zamm and colleagues (Zamm et al. 2013; Neufeld et al. 2012) reported stronger anatomical connections between visual and auditory areas as indexed by DTI measures in colored-hearing synesthetes. Especially the inferior frontooccipital fasciculus (IFOF) on the right was larger in colored-hearing synesthetes than in controls. Using fMRI and functional connectivity measures measured during a double-flash illusion ${ }^{2}$ Neufeld and colleagues (Neufeld et al. 2012) revealed no generally enhanced functional coupling between auditory and visual areas in synesthetes of the same type. They identified, however, an increased coupling between the inferior parietal lobule (IPL) and the auditory cortex, which they interpret as indicator of a stronger disinhibition of the auditory cortex from the IPL. However, whether this stronger coupling from the IPL to the auditory cortex indicated stronger inhibition has to be confirmed in future studies. It is also possible that this coupling indicates an intimate functional relationship between the IPL and the auditory cortex supporting binding and thus multimodal integration processes since the IPL has been shown to be strongly involved in these processes. These studies emphasize the role of the functional and anatomical coupling between distant brain areas in synesthesia. An extreme position, in this context, has been offered by Hupe et al. (2011). They identified rather distributed specific anatomic features outside the color areas. Especially the retrosplenial area turned out to demonstrate more white matter (WM) in grapheme-color synesthetes. Thus, they conclude that "the joint coding of real and synesthetic colors must, therefore, be distributed rather than localized in the visual cortex".

A recent paper of our group emphasizes the specific role of anatomical network features in the synesthete's brain (Hanggi et al. 2011). Using cortical thickness measures obtained from structural MRI measurements of the brain of synesthetes, we estimated to cortical connectivity on the

\footnotetext{
2 This illusion is induced by a single light flash presented together with multiple beep sounds, which is then perceived as multiple flashes. This illusion is a helpful technique to study audio-visual integration.
} 
basis of graph-theoretical techniques. As a result, we identified that synesthetes (here we studied grapheme-color synesthetes) showed increased whole-brain interconnectivity compared to non-synesthetes. Thus not only the brain areas known to be involved in controlling synesthetic experiences are strongly interconnected but also other brain which have not been identified as being specific for synesthetic experiences. Based on these findings, we assume that the synesthetic brain is characterized by a kind of overconnectivity.

In a previous paper, we identified a kind of focal overconnectivity for our famous synesthetes E.S. who is a toneinterval taste synesthetes (Hanggi et al. 2008). She experiences particular tastes on the tongue when listening to particular tone intervals. This extraordinary synesthesia is related to a kind of over-connectivity in the auditory cortex extending into the insula. This over-connectivity can possibly the reason why E.S. experiences tastes as a consequence of listening to tone intervals because the auditory cortex is so strongly and tightly connected with the insula housing neural networks known to be involved in taste perception.

Taken together, the brain of synesthetes demonstrates lots of anatomical and functional differences compared to non-synesthetes. These differences support the idea of a highly individual brain where specific abilities are linked to specific anatomical and functional features.

\section{Summary and conclusion}

Synesthesia is a fascinating phenomenon, which now attracts many neuroscientists and cognitive psychologists all over the world. The recent research within the last 10-15 years has uncovered several interesting findings increasing the understanding of synesthetic experiences. Currently, three models are used to explain synesthesia: (1) the two-stage cross-activation and hyper-binding model, (2) the disinhibited feedback model, and (3) the limbicmediation model. Which of these models sufficiently explain synesthesia has to be shown in future experiments. However, several neurophysiological studies provide support for at least two of these models (the cross-activation and hyper-binding as well as the disinhibited feedback model). An interesting issue of modern synesthesia research is that synesthesia is most likely due to different psychological processes, which might interact. These different processes might also be helpful to explain interindividual differences between synesthetes, e.g., those who rely more strongly on different perceptual (or bottom-up) processes and those who rely more strongly on cognitive (top-down) processes (following the distinction in "lower" and "higher" synesthetes).
While earlier neuroscience studies have focused more on local anatomical and neurophysiological features of the brain areas involved in processing the synesthetic experience, there is currently a shift to a more network-oriented approach to explain the neural underpinnings of the interesting phenomenon. These new studies have also refocused the interest into the dynamic functional coupling between the involved neural assemblies.

There is also a growing interest to examine whether synesthesia is associated with benefits in cognitive processing. More and more studies demonstrate that synesthesia is indeed associated with cognitive benefits. For example, it has been shown that synesthetes use their unusual experience as mnemonic device, are superior in long-term memory, and demonstrate a different cognitive style (Watson et al. 2014; Meier and Rothen 2013).

A further interesting question is whether synesthesia is genetically driven and a kind of trait endowing only a few subjects to perceive the world in the synesthetic specific way or whether it could be learned or at least modified by experience. Some studies indeed support the idea that synesthesia is at least partly influenced by experience. For example Beeli et al. (2007) showed that the concurrent color perceptions in grapheme-color synesthetes are related to the frequency of occurrence of the graphemes in the lexicon. That experience must have a substantial influence on synesthetic experiences has also been implicated by Beeli et al. report of a tone interval to taste synesthete (2005). This synesthete reports particular taste perceptions to the perceptions of particular tone intervals. Since this synesthete learned the tone intervals when she started musical practice at the age of 5-6 years this form of synesthesia has most likely been influenced by musical practice. For future studies, it would be fascinating to examine whether synesthetic experiences can be established in adulthood even in subjects who never have experienced synesthesias before. In this context, it would also be interesting to study whether an acquired synesthesia might influence the personality, attitudes, or even interests of these subjects.

\section{References}

Barnett KJ, Foxe JJ, Molholm S, Kelly SP, Shalgi S, Mitchell KJ et al (2008a) Differences in early sensory-perceptual processing in synesthesia: a visual evoked potential study. Neuroimage 43:605-613

Barnett KJ, Finucane C, Asher JE, Bargary G, Corvin AP, Newell FN et al (2008b) Familial patterns and the origins of individual differences in synaesthesia. Cognition 106(2):871-893

Baron-Cohen S, Burt L, Smith-Laittan F, Harrison J, Bolton P (1996) Synaesthesia: prevalence and familiality. Perception 25(9):1073-1079

Beeli G, Esslen M, Jancke L (2005) Synaesthesia: when coloured sounds taste sweet. Nature 434:38 
Beeli G, Esslen M, Jäncke L (2007) Frequency correlates in grapheme-color synaesthesia. Psychol Sci 18:788-792

Beeli G, Esslen M, Jäncke L (2008) Time course of neural activity correlated with colored-hearing synesthesia. Cereb Cortex 18:379-385

Bleuler E, Lehmann K (1871) Zwangsmässige Lichtempfindungen durch Schall und verwandte Erscheinungen auf dem Gebiete der andern Sinnesempfindungen. Fues's, Leipzig

Brang D, Ramachandran VS (2011) Survival of the synesthesia gene: why do people hear colors and taste words? PLoS Biol 9(11):e1001205 NeuroImage, 53, 268-274

Brang D, Edwards L, Ramachandran VS, Coulson S (2008) Is the sky 2? Contextual priming in grapheme-color synaesthesia. Psychol Sci 19:421-428

Brang D, Hubbard EM, Coulson S, Huang M, Ramachandran VS (2010) Magnetoencephalography reveals early activation of V4 in grapheme-color synesthesia. Neuroimage 53(1):268-274

Bunzeck N, Wuestenberg T, Lutz K, Heinze HJ, Jancke L (2005) Scanning silence: mental imagery of complex sounds. Neuroimage 26:1119-1127

Campen Cvv (2010) The Hidden Sense: Synesthesia in Art and Science (Leonardo Book Series) (Reprint ed.). The MIT Press

Cohen Kadosh R, Cohen Kadosh K, Henik A (2007) The neuronal correlate of bidirectional synesthesia: a combined event-related potential and functional magnetic resonance imaging study. J Cogn Neurosci 19:2050-2059

Cytowic RE, Wood FB (1982a) Synesthesia. I. A review of major theories and their brain basis. Brain Cogn 1:23-35

Cytowic RE, Wood FB (1982b) Synesthesia. II. Psychophysical relations in the synesthesia of geometrically shaped taste and colored hearing. Brain Cogn 1:36-49

Elias LJ, Saucier DM, Hardie C, Sarty GE (2003) Dissociating semantic and perceptual components of synaesthesia: behavioural and functional neuroanatomical investigations. Cogn Brain Res 16:232-237

Gebuis T, Nijboer TCW, Van der Smagt MJ (2009) Multiple dimensions in bi-directional synesthesia. Eur $\mathrm{J}$ Neurosci 29:1703-1710

Goller AI, Otten LJ, Ward J (2009) Seeing sounds and hearing colors: an event-related potential study of auditory-visual synesthesia. J Cogn Neurosci 21:1869-1881

Grossenbacher PG, Lovelace CT (2001) Mechanisms of synesthesia: cognitive and physiological constraints. Trends Cogn Sci 5:36-41

Hanggi J, Beeli G, Oechslin MS, Jancke L (2008) The multiple synaesthete E.S.: neuroanatomical basis of interval-taste and tone-colour synaesthesia. Neuroimage 43:192-203

Hanggi J, Wotruba D, Jancke L (2011) Globally altered structural brain network topology in grapheme-color synesthesia. J Neurosci 31:5816-5828

Hubbard EM (2007) Neurophysiology of synesthesia. Curr Psychiatry Rep 9:193-199

Hubbard EM, Arman AC, Ramachandran VS, Boynton GM (2005) Individual differences among grapheme-color synesthetes: brainbehavior correlations. Neuron 45:975-985

Hupe JM, Bordier C, Dojat M (2011) The neural bases of graphemecolor synesthesia are not localized in real color-sensitive areas. Cereb Cortex 22:1622-1633

Jäncke L (2013) The timing of neurophysiological events in synaesthesia. In: Simner J, Hubbard EM (eds) Oxford handbook of synesthesia. Oxford University Press, Oxford

Jäncke L, Langer N (2011) A strong parietal hub in the small-world network of coloured-hearing synaesthetes during resting state EEG. J Neuropsychol 5:178-202

Jäncke L, Shah NJ (2004) Hearing syllables by seeing visual stimuli. Eur J Neurosci 19:2603-2608
Jäncke L, Rogenmoser L, Meyer M, Elmer S (2012) Pre-attentive modulation of brain responses to tones in coloured-hearing synesthetes. BMC Neurosci 13:151

Knoch D, Gianotti LR, Mohr C, Brugger P (2005) Synesthesia: when colors count. Cogn Brain Res 25:372-374

Meier B, Rothen N (2007) When conditioned responses "fire back": bidirectional cross-activation creates learning opportunities in synesthesia. Neuroscience 147:569-572

Meier B, Rothen N (2013) Grapheme-color synaesthesia is associated with a distinct cognitive style. Front Psychol 4:1-7

Neufeld J, Sinke C, Zedler M, Emrich HM, Szycik GR (2012) Reduced audio-visual integration in synaesthetes indicated by the double-flash illusion. Brain Res 1473:78-86

Niccolai V, Jennes J, Stoerig P, Van Leeuwen TM (2012a) Modality and variability of synesthetic experience. Am $\mathrm{J}$ Psychol 125(1):81-94

Niccolai V, Wascher E, Stoerig P (2012b) Distinct neural processes in grapheme-colour synaesthetes and semantic controls. Eur J Neurosci 36:1-9

Nunn JA, Gregory LJ, Brammer M, Williams SCR, Parslow DM, Morgan MJ et al (2002) Functional magnetic resonance imaging of synesthesia: activation of V4/V8 by spoken words. Nat Neurosci 5:371-375

Oechslin MS, Meyer M, Jäncke L (2010) Absolute pitch-functional evidence of speech-relevant auditory acuity. Cereb Cortex 20:447-455

Paulesu E, Harrison J, Baron-Cohen S, Watson JD, Goldstein L, Heather J et al (1995) The physiology of coloured hearing. A PET activation study of colour-word synaesthesia. Brain 118:661-676

Proverbio AM, D'Aniello GE, Adorni R, Zani A (2011) When a photograph can be heard: vision activates the auditory cortex within $110 \mathrm{~ms}$. Sci Rep 1:54

Ramachandran VS, Hubbard EM (2001) Psychophysical investigations into the neural basis of synaesthesia. Proc Royal Soc Lond Ser B Biol Sci 268:979-983

Rouw R, Scholte HS, Colizoli O (2011) Brain areas involved in synaesthesia: a review. J Neuropsychol 5:214-242

Schiltz K, Trocha K, Wieringa BM, Emrich HM, Johannes S, Munte TF (1999) Neurophysiological aspects of synesthetic experience. J Neuropsychiatry Clin Neurosci 11:58-65

Simner J (2012) Defining synaesthesia: a response to two excellent commentaries. Br J Psychol 103(1):24-27

Sinke C, Neufeld J, Wiswede D, Emrich HM, Bleich S, Munte TF et al (2014) N1 enhancement in synesthesia during visual and audio-visual perception in semantic cross-modal conflict situations: an ERP study. Front Hum Neurosci 8:21

Smilek D, Dixon MJ, Cudahy C, Merikle PM (2001) Synaesthetic photisms influence visual perception. J Cogn Neurosci 13:930-936

Smilek D, Dixon MJ, Cudahy C, Merikle PM (2002) Concept driven color experiences in digit-color synesthesia. Brain Cogn 48:570-573

Smilek D, Dixon MJ, Merikle PM (2005) Synaesthesia: discordant male monozygotic twins. Neurocase 11:363-370

Teuscher U, Brang D, Ramachandran VS, Coulson S (2010) Spatial cueing in time-space synesthetes: an event-related brain potential study. Brain Cogn 74:35-46

van Leeuwen TM, Petersson KM, Hagoort P (2010) Synaesthetic colour in the brain: beyond colour areas. A functional magnetic resonance imaging study of synaesthetes and matched controls. PLoS One 5:e12074

Volberg G, Karmann A, Birkner S, Greenlee MW (2013) Short- and long-range neural synchrony in grapheme-color synesthesia. J Cogn Neurosci 25(7):1148-1162 
Vulpian EFA (1866) Leçons sur la physiologie générale et comparée du système nerveux, faites en 1864 au Muséum d'histoire naturelle. Gerner-Baillière, Paris

Watson MR, Akins KA, Spiker C, Crawford L, Enns JT (2014) Synesthesia and learning: a critical review and novel theory. Front Hum Neurosci 8:98

Weiss PH, Shah NJ, Toni I, Zilles K, Fink GR (2001) Associating colours with people: a case of chromatic-lexical synaesthesia. Cortex 37:750-753
Weiss PH, Zilles K, Fink GR (2005) When visual perception causes feeling: enhanced cross-modal processing in grapheme-color synesthesia. Neuroimage 28:859-868

Weiss PH, Kalckert A, Fink GR (2009) Priming letters by colors: evidence for the bidirectionality of grapheme-color synesthesia. J Cogn Neurosci 21:2019-2026

Zamm A, Schlaug G, Eagleman DM, Loui P (2013) Pathways to seeing music: enhanced structural connectivity in colored-music synesthesia. Neuroimage 74:359-366 